\title{
Distinguishing sources of pain: Central vs peripheral mediation
}

\author{
(1) RICHARD GEVIRTZ
}

Department of Clinical Psychology, CSPP Alliant International University, San Diego, USA

\begin{abstract}
Research in chronic pain often fails to distinguish the pain source: central vs peripheral. In this review, I lay out a case for a greater consideration of a peripheral pain source, namely myofascial trigger points. Findings from our group are presented indicating that trigger points are alpha sympathetically innervated and are not directly related to the cholinergic neuro-muscular system. Treatment implications are discussed.
\end{abstract}

Keywords: Myofascial pain trigger points; sympathetic muscle innervation

\section{INTRODUCTION}

Chronic pain, although difficult to define, is now clearly recognized as a major health problem creating a worldwide burden. ${ }^{1}$ It is useful to classify chronic pain as having a primarily central vs a peripheral etiology. Clear cut examples of centrally mediated pain are phantom limb pain, Fibromyalgia (allodynia and hyperalgesia), and perhaps Complex Regional Pain Syndromes. A great deal of work has been published to try and elucidate the neural, behavioral and cognitive aspects of centrally mediated pain. An excellent recent review tries to model brain plasticity in brain organization for these pain syndromes. ${ }^{2}$

"A large body of converging evidence suggests that chronic pain is not simply a temporal extension of acute pain but involves distinct mechanisms. The transition of acute pain into a chronic disorder involves activity dependent changes (that is, functional plasticity) at many different interconnected levels, ranging from the molecular to the network level, at several anatomical avenues in the nociceptive pathway., ${ }^{3,4}$ This interconnectivity can explain why even small molecular changes, such as a single point mutation, can result in large changes at the behavioural or clinical levels that are caused by amplification along multiple scales of plasticity. Mechanisms involving functional plasticity have been studied extensively and have revealed a range of modulatory factors that change the sensory, emotional and cognitive components of pain (reviewed in. ${ }^{5-8}$ However, recent data show that functional plasticity changes are accompanied by structural re-modelling and reorganization of synapses, cells and circuits that can also occur at various anatomical and temporal scales ${ }^{8,9}$ thereby further adding complexity and a large dynamic range, and potentially accounting for the development of pain that extends over longer periods of time. Structural re-modelling of connections has not been studied as widely as functional plasticity, and it remains unclear whether it represents a cause or a consequence of chronic pain"2(p.20).

While this perspective is clearly informative and relevant to pain syndromes involving allodynia or hyperalgesia, it may be missing a large number of chronic pain disorders that may have a peripheral source. In this paper, I would like to focus on myofascial or muscular pain, which appears to constitute the largest number of chronic pain patients ${ }^{10,11}$ and may be relatively independent of the central sensitization discussed above. 
Our group has struggled with the lack of a clear mediational model connecting psychological stress and muscle pain (Diagram 1). There seem little doubt that psychological/emotional features play a role in pain, but other than postulating a central nervous system pain amplification system, little was known about this "mind to muscle connection". Naîve theories postulated that stress caused striate muscle groups to contract chronically, thus producing overuse and pain. The problem here is that there is no evidence that painful muscles have byproducts associated with pain, nor are they chronically contracted [based on surface electromyogram (EMG) studies]. There is some indication that a fascial contracture may occur, but this new line of research has not been widely recognized..$^{12}$ In fact, an exhaustive search for a peripheral biomarker associated with chronic pain, has turned up no meaningful answers.

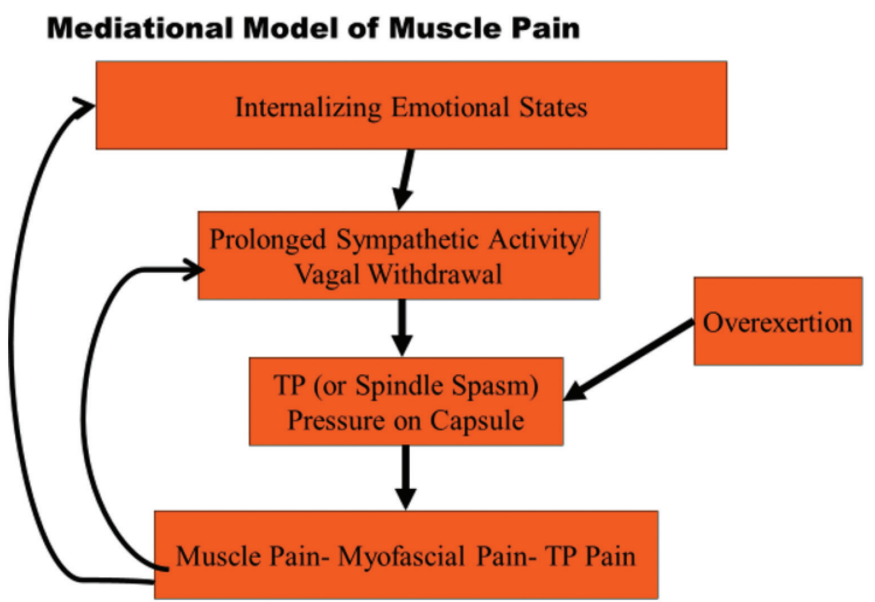

Diagram 1. Mediational model of Muscle pain TP: Trigger point

Increasingly, pain from local nodules called trigger points has emerged as a promising source of muscle pain syndromes such as tension headache, neck pain, low back pain, pelvic floor pain, etc.

Shah et al. ${ }^{13}$ provide a detailed historical perspective on myofascial or trigger point pain. While the concept of myofascial trigger points (MTrPs) can be traced back to Guillaume de Baillous (1538-1616) of France, the pioneering work of Janet Travell marks the coming of age of this pain conceptualization. ${ }^{14,15}$ Travell et al. ${ }^{15}$ catalogued the location of MTrPs throughout the body in great detail in two volumes of their handbook. Their exhaustive analyses are widely accepted as accurate. While the underlying physiology of MTrPs is quite controversial, a number of features are widely accepted: Associated stiffness
Localized point tenderness in muscle

Stimulation produces local and referred pain Often with a palpable taut band

Twitch

Trigger because like a gun trigger is initiated with pressure Produces pain in another place-(target).

While the myofascial perspective is now widely known as applying to tension headache, neck pain, upper body pain, lower back pain, etc., recent work has focused on pelvic floor pain. Jantos et al. ${ }^{17}$ have described in detail a peripheral pain model that incorporates the myofascial perspective. ${ }^{16-18}$

Thus, MTrPs represent a peripheral source of pain, at least partially, and I would argue, substantially independent of central sensitization. For this reason, more emphasis on these pain syndromes is warranted.

\section{MATERIALS AND METHODS}

\section{Mediational model}

Our group began investigating MTrPs about 20 years ago. We began by trying to find a biological signature using surface electromyographic methods. This approach proved unfruitful. Needle electrodes were then proposed, but where should the needle be inserted in the muscle? A medical student (Dawn Bravata), working with us on various projects suggested a trigger point in the trapezius muscle. At that time Travell's work ${ }^{19}$ was not well known nor accepted in medical circles, so we were quite skeptical at this suggestion. But in a rare fit of open-mindedness we tried inserting the needle into a tender nodule in the trapezius and —Eureka, - - the needle EMG (nEMG) monitor lit up at a very specific locale. A second needle nearby in non-tender tissue remained electrically silent. Figure 1 shows a typical finding.

This method was then perfected and the first systematic study run and published. ${ }^{19} \mathrm{Almost}$ all of the subsequent studies used this methodology. We estimate that we have tested over 300 patients in this manner. With rare exception, we get the same pattern.

A series of pharmacological studies followed ${ }^{20}$ that showed that the $\mathrm{nEMG}$ activity in the trigger point (TP) was unaffected by curare (a powerful cholinergic blocker that blocks all motor neuron activity), but dampened by phentolamine (an alphasympathetic blocker, see Figure 2). Unaffected by acetylcholine, the usual motor neuron neuro-transmitter, but blocked by a sympathetic blocker, this work seemed to offer a hypothesis about the pathways for sympathetically mediated peripheral pain in muscle. 


\section{Needle EMG Activity}

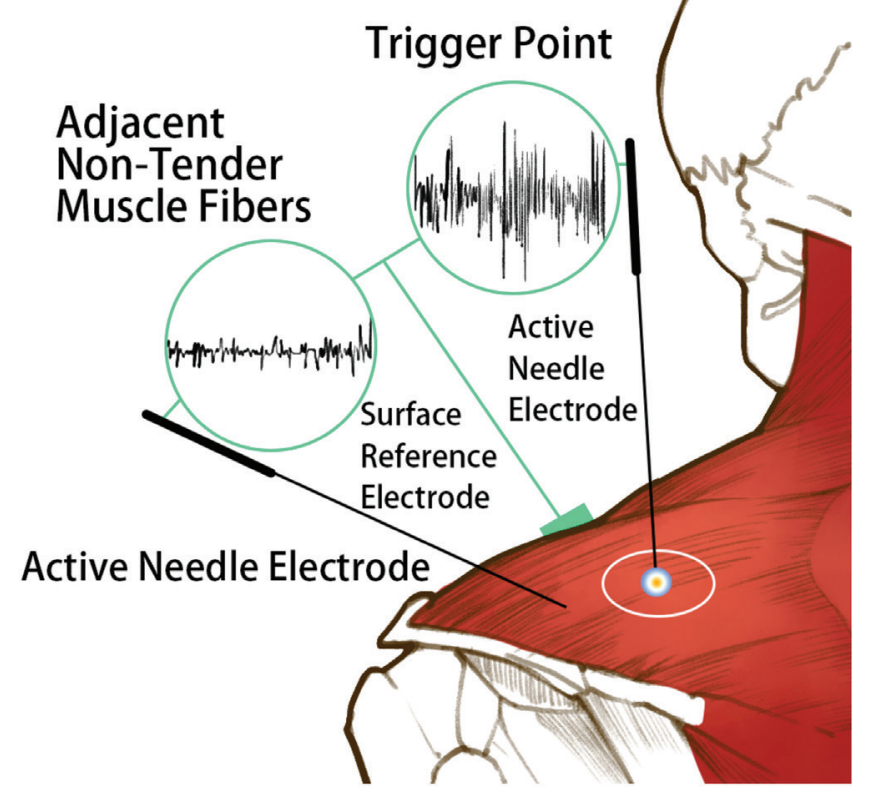

Figure 1. Needle EMG ( $\mathrm{nEMG}$ ) paradigm. Left circle shows activity in the non-tender site, right circle for the trigger point EMG: Electromyogram

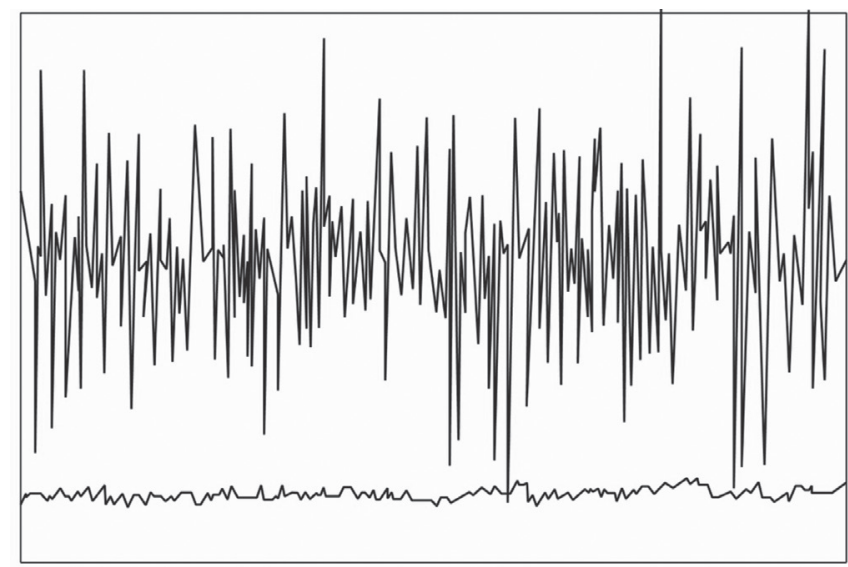

Figure 2. It shows the $\mathrm{nEMG}$ action potentials for Curare, lower is the non-tender adjacent site nEMG: Neddle electromyogram

We then began a series of psychophysiological studies. My student, McNulty et al. ${ }^{21}$, gathered subjects with palpable TPs and found that the TP, but not the adjacent site responded vigorously to psychological stress. As our theory predicted, the non-tender site remained silent during the stress. It seems that the muscle contraction we see in the clinic during stress can be inhibited with instruction (and perhaps the fear of moving a needle in the muscle). Another student, Banks ${ }^{22}$ replicated the
McNulty paradigm but added a relaxation component. TPs were clearly activated during stress but activation decreased during Autogenic Relaxation. Lewis et al. ${ }^{23}$ found the same relationships existed in pain patients.

Since we were unsure of the nature of the psychological stimuli that would invoke a TP response, we then undertook a series of studies to elucidate the emotional stimuli. Gadler et al..$^{24}$ used interview techniques to try and elicit TP responses and found that during recall, very high nEMG activity was produced (up to 120 micro-volts). Professional actors were used by Cafaro ${ }^{25}$ to see if dramatically expressed or inhibited emotions would produce higher TP activity. They didn't; it seems that recall of an emotional event drives the TPs at about the same rate as an angry or volatile outburst.

\section{RESULTS}

Based on these results, we postulate a mediational model of chronic muscle pain that yields potential treatment models. Figure 3 illustrates the broad model. It is hypothesized that chronic muscle pain syndromes result from activation of MTrPs by overstretch, emotional stress, or other sources that create a powerful afferent signal to pain perception centers in the brain. Thus, chronic sources of stress such as anxiety, worry, or other internalizing emotional states can fuel activity in the MTrP.

We have postulated that the MTrPs are actually muscle spindles (the intrafusal fibers within capsules responsible for stretch perception and regulation). This idea has been challenged, but is worthy of further exploration. ${ }^{13}$

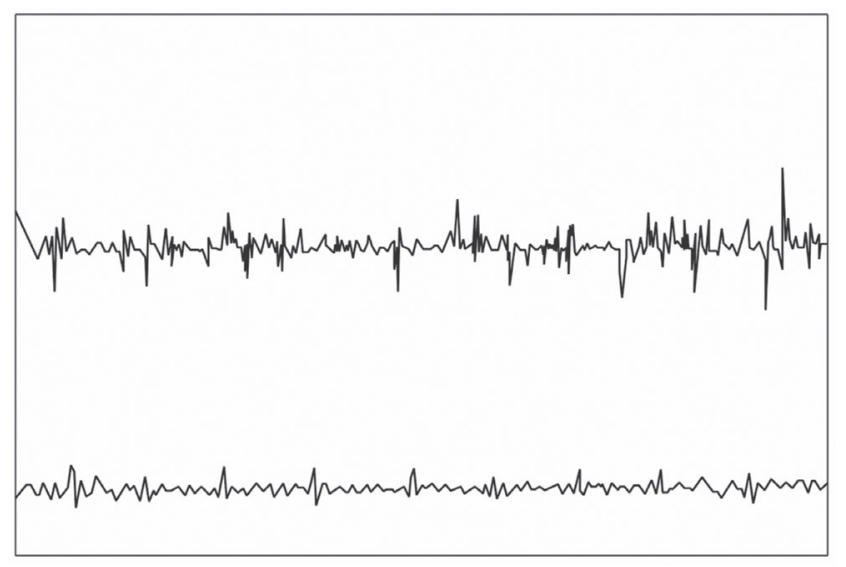

Figure 3. It shows the nEMG action potentials for Phentolamine, lower is the non-tender adjacent site nEMG: Neddle electromyogram 


\section{DISCUSSION}

\section{Treatment models}

It has long been recognized that techniques such as acupressure, massage, or dry needling are effective in reducing pain in MTrPs, at least temporarily (c.f. $)^{26,27}$ Longer term relief has been a more elusive goal. Based on the above model, we have postulated that combining techniques that reduce sympathetic outflow with manual release techniques might offer longer term relief. One such technique, that has gained increasing support is Heart Rate Variability Biofeedback (HRVB).

In this protocol, patients learn to breathe at a specific rate diaphragmatically so as to greatly increase beat to beat heart rate accelerations during inhalation and decelerations during exhalations. Regular practice at this resonance frequency increases autonomic flexibility such that the vagus can limit sympathetic outflow to various target organs. ${ }^{28-31}$ By combining this type biofeedback-based home practice technique, we have found that pain relief is prolonged dramatically, presumably because re-stimulation of the MTrPs is limited by better autonomic balance and a more robust parasympathetic (vagal) tone. ${ }^{32-34}$

The rapid response to the above treatment protocols seems to imply that central sensitization plays a relatively minor role in these syndromes, as compared to Fibromyalgia for example.

\section{CONCLUSIONS}

The growing recognition of the burden of chronic pain requires complex analyses that recognize the sources of pain. It is argued here, that recognizing the important role of peripheral sources of pain, specifically MTrPs, can greatly enhance treatment models so as to reduce pain, restore functionality, and reduce treatment costs.

\section{DISCLOSURES}

The authors declare no conflict of interest, and no financial support by any grant or research sponsor.

Peer-review: Externally peer-reviewed.

\section{REFERENCES}

1. Raffaeli W, Arnaudo E. Pain as a disease: an overview. J Pain Res 2017; 10: 2003-8.

2. Kuner R, Flor H. Structural plasticity and reorganisation in chronic pain. Nature Rev Neurosci 2017; 18: 113.

3. Prescott SA, Ma Q, De Koninck Y. Normal and abnormal coding of somatosensory stimuli causing pain. Nat Neurosci 2014; 17: 183-91.

4. Sandkühler J. Models and mechanisms of hyperalgesia and allodynia. Physiol Rev 2009; 89: 707-58.
5. Baliki MN, Baria AT, Apkarian AV. The cortical rhythms of chronic back pain. J Neurosci 2011; 31: 13981-90.

6. Basbaum Al, Bautista DM, Scherrer G, Julius D. Cellular and molecular mechanisms of pain. Cell 2009; 139: 267-84.

7. Ji RR, Berta T, Nedergaard M. Glia and pain: is chronic pain a gliopathy? Pain 2013; 154(Suppl 1): 10-28.

8. Kuner R. Central mechanisms of pathological pain. Nat Med 2010; 16: 1258-66.

9. Flor H, Nikolajsen L, Staehelin Jensen T. Phantom limb pain: a case of maladaptive CNS plasticity? Nat Rev Neurosci 2006; 7: 873-81.

10. Skootsky SA, Jaeger B, Oye RK. Prevalence of myofascial pain in general internal medicine practice. West J Med 1989; 151: 157-60.

11. Gerwin RD. Classification, epidemiology, and natural history of myofascial pain syndrome. Curr Pain Headache Rep 2001; 5: 412 20.

12. Schleip R, Klingler W. Active contractile properties of fascia. Clin Anat 2019; 32: 891-5.

13. Shah JP, Thaker N, Heimur J, Aredo JV, Sikdar S, Gerber L. Myofascial trigger points then and now: a historical and scientific perspective. PM\&R, 2015; 7: 746-61.

14. Travell J, Rinzler SH. The myofascial genesis of pain. Postgrad Med 1952; 11:434-52.

15. Travell JG, Simons DG. Myofascial pain and dysfunction: the trigger point manual. Williams \& Wilkins; Baltimore, 1983.

16. Jantos M, Johns S, Torres A, Radomanska EB. Mapping chronic urogenital pain in women: insights into mechanisms and management of pain based on the IMAP, Part 2. Pelviperineology 2015; 34: 28-36.

17. Jantos M, Johns S, Torres A, Radomanska EB. Mapping chronic urogenital pain in women: review and rationale for a muscle assessment protocol - Part 1. Pelviperineology 2015; 34: 21-7.

18. Johns S, Jantos M, Baszak-Radomańska E. Refining a Pain Mapping Tool. J Low Genit Tract Dis 2017; 21(Suppl 4): 10.

19. Hubbard DR, Berkoff GM. Myofascial trigger points show spontaneous needle EMG activity. Spine (Phila Pa 1976) 1993; 18 : 1803-7.

20. Hubbard D. Chronic and Recurrent Muscle Pain: Pathophysiology and treatment, a review of pharmocologic studies. Journal of Musculoskelatal Pain 1996; 4: 123-44.

21. McNulty WH, Gevirtz RN, Hubbard DR, Berkoff GM. Needle electromyographic evaluation of trigger point response to a psychological stressor. Psychophysiology 1994; 31: 313-6.

22. Banks SL, Jacobs DW, Gevirtz RJ, Hubbard DR. Effects of autogenic relaxation training on electromyographic activity in active myofascial trigger points. Journal of Musculoskelatal Pain 1998; 64: 23-32.

23. Lewis C, Gevirtz R, Hubbard D, Berkoff G. Needle trigger point and surface frontal EMG measurements of psychophysiological responses in tension-type headache patients. In Biofeedback and Self-Regulation. New York NY: Spring ST, 1994; 19: 274-5.

24. Gadler R, Gevirtz RN. Evaluation of Needle Electromyographic Response to Emotional Stimuli. Applied Psychophysiology and Biofeedback, 1997; 22: 137. 
25. Cafaro TA, Gevirtz RN, Hubbard D, Harvey M. The exploration of trigger point and heart rate variability excitation and recovery patterns in actors performing anger inhibition and anger expression. Applied Psychophysiology and Biofeedback, 2001; 26: 236.

26. Moraska AF, Schmiege SJ, Mann JD, Butryn N, Krutsch JP. Responsiveness of Myofascial Trigger Points to Single and Multiple Trigger Point Release Massages: A Randomized, Placebo Controlled Trial. Am J Phys Med Rehabil 2017; 96: 639-45.

27. Charles D, Hudgins T, MacNaughton J, Newman E, Tan J, Wigger M. A systematic review of manual therapy techniques, dry cupping and dry needling in the reduction of myofascial pain and myofascial trigger points. J Bodyw Mov Ther 2019; 23: 539-46.

28. Gevirtz RN, Lehrer PM, Schwartz MS. Cardiorespiratory biofeedback. Biofeedback: A Practitioner's Guide, 2016: 196-213.

29. Lehrer PM, Gevirtz R. Heart rate variability biofeedback: how and why does it work? Front Psychol 2014; 21: 756.

30. Gevirtz R. The promise of heart rate variability biofeedback: Evidence-based applications. Biofeedback, 2013; 41: 110-20.
31. Gevirtz R, Hubbard D, Harpin E. Psychophysiologic Treatment of Chronic Low Back Pain. Professional Psychology: Research and Practice, 1996; 27: 561-6.

32. Hautzinger M. Myofascial release in combination with trigger point therapy and deep breathing training improves low back pain. In Fascia Research II, Basic Science and Implications for Conventional and Complementary Health Care. Elsevier, 2009: 249.

33. Vagedes J, Gordon CM, Schwaemmle M, et al. Does Deep Breathing Training Improve Myofascial Release in Combination with Trigger Point Therapy for Patients with Low Back Pain?. Applied Psychophysiology and Biofeedback, 2011; 36: 295.

34. Vagedes J, Gordon CM, Beutinger D, et al. Myofascial release in combination with trigger point therapy and deep breathing training improves low back pain. In: Fascia Research II, Basic Science and Implications for Conventional and Complementary Health Care. Elsevier, 2009: 249. 